\title{
EULER AND NAVIER-STOKES EQUATIONS
}

\author{
Peter Constantin
}

Abstract

We present results concerning the local existence, regularity and possible blow up of solutions to incompressible Euler and NavierStokes equations.

\section{Contents}

1. Introduction 235

2. Euler Equations 236

3. The Eulerian-Lagrangian Description 237

4. Local Existence 241

5. The Blow Up Issue for Euler Equations 247

6. Navier-Stokes Equations 253

7. The Blow Up Issue for Navier-Stokes Equations 256

References $\quad 261$

\section{Introduction}

The notes below present some of the mathematical problems concerning the equations of ideal incompressible fluids (Euler equations) and viscous incompressible fluids (Navier-Stokes equations). The approach used is an Eulerian-Lagrangian description based on the "back-to-labels" map. In the case of the Euler equations, this map is the inverse Lagrangian path map; in the case of the Navier-Stokes equations, it is a diffusive analogue. The notes present local existence results and discuss some of the blow up issues, separately for ideal and viscous flows.

2000 Mathematics Subject Classification. 76B03, 76D05.

Key words. Euler equations, Navier-Stokes equations, Eulerian-Lagrangian description. 


\section{Euler Equations}

The three dimensional Euler equations describe ideal, frictionless fluids. They are evolution equations for the three velocity components $u(x, t)$,

$$
\frac{\partial u}{\partial t}+u \cdot \nabla u+\nabla p=0,
$$

coupled with a fourth equation,

$$
\nabla \cdot u=0,
$$

which is the incompressibility constraint. This is the Eulerian formulation with $x \in \mathbb{R}^{3}, t \in \mathbb{R}$. The pressure $p$ is determined by imposing the incompressibility,

$$
-\Delta p=\nabla \cdot(u \cdot \nabla u) .
$$

There are no natural boundary conditions for this equation, except when the fluid occupies all of $\mathbb{R}^{3}$ (decay at infinity) or when space-periodic boundary conditions are imposed. In either one of these cases, the pressure is determined up to an additive constant. The Euler equations are conservative: The total kinetic energy,

$$
\int|u|^{2} d x
$$

is a constant of motion. Some reference books on Euler equations are [1] $[\mathbf{8}],[\mathbf{4 4}]$ and on turbulence $[\mathbf{1 0}],[\mathbf{3 9}]$. The circulation around a closed curve $\gamma$,

$$
\oint_{\gamma} u \cdot d x
$$

is time independent, if the curve moves with the flow. This important conservation law has not yet found its rightful role in the mathematical analysis. The vorticity $\omega=\nabla \times u$ describes the rotation in the fluid. It obeys a quadratic equation, whose nature is such that the magnitude of the vorticity might increase in time. The vorticity equation is equivalent to the vanishing of the commutator

$$
\left[D_{t}, \omega \cdot \nabla\right]=0
$$

where

$$
D_{t}=\frac{\partial}{\partial t}+u \cdot \nabla
$$

is the material derivative (derivative along flow paths). The characteristics of the first order differential operator $\omega \cdot \nabla$ are called vortex lines; the characteristics of the material derivative $D_{t}$ are Lagrangian particle 
paths. The vanishing of the commutator means that vortex lines are carried by the flow. The flow is the path map $a \mapsto X(a, t)$. The connection between the Lagrangian description and the Eulerian one is given by the relations

$$
u(x, t)=\frac{\partial X(a, t)}{\partial t}, \quad x=X(a, t) .
$$

The initial value problem for the Euler equations is to solve (1), (2) with given initial velocity $u_{(0)}$. This problem is known to have a solution for smooth enough initial data and short enough time $([\mathbf{3 4}],[\mathbf{4 1}])$.

We discuss a description of the Euler equations as a system of three coupled active scalar equations. Active scalar equations have the form

$$
D_{t} \theta=0,
$$

with $u=U[\theta]$, where $U$ is a time-independent functional of $\theta$. The functional need not be linear, and, in general, it is not local. The description of the Euler equations concerns Lagrangian quantities computed in Eulerian variables and is based on the Weber formula (18) ([53]) that represents the velocity in terms of the inverse of the Lagrangian map.

\section{The Eulerian-Lagrangian Description}

The Lagrangian formulation of the Euler equations is a second order, non-local evolution equation for the map $a \mapsto X(a, t)$. The curve $t \mapsto$ $X(a, t)$ is the Lagrangian path at label $a$ and obeys Newton's law

$$
\frac{\partial^{2} X(a, t)}{\partial t^{2}}=F_{X}(a, t)
$$

The incompressibility is expressed as the volume-preserving condition for the map:

$$
\operatorname{det}\left(\nabla_{a} X\right)=1
$$

The initial condition sets the labels at the initial time,

$$
X(a, 0)=a .
$$

The forces $F_{X}$ in $(5)$ are conservative:

$$
F_{X}(a, t)=-\left(\nabla_{x} p\right)(X(a, t))=-\left[\left(\nabla_{a} X(a, t)\right)^{*}\right]^{-1}\left(\nabla_{a} \tilde{p}\right)(a, t)
$$

with $\tilde{p}(a, t)=p(X(a, t))$ and where $p$ is the Eulerian pressure. The notation $M^{*}$ means the transpose of the matrix $M,\left(M^{*}\right)^{-1}$ its inverse. 
We show now a quick proof of the Weber formula. Multiplying (5) by $\left(\nabla_{a} X\right)^{*}$ we obtain

$$
\left(\nabla_{a} X(a, t)\right)^{*} \frac{\partial^{2} X(a, t)}{\partial t^{2}}=-\left(\nabla_{a} \tilde{p}\right)(a, t)
$$

that is,

$$
\frac{\partial^{2} X^{j}(a, t)}{\partial t^{2}} \frac{\partial X^{j}(a, t)}{\partial a_{i}}=-\frac{\partial \tilde{p}(a, t)}{\partial a_{i}} .
$$

Pulling out a time derivative in the left-hand side we obtain

$$
\frac{\partial}{\partial t}\left[\frac{\partial X^{j}(a, t)}{\partial t} \frac{\partial X^{j}(a, t)}{\partial a_{i}}\right]=-\frac{\partial \tilde{q}(a, t)}{\partial a_{i}}
$$

where

$$
\tilde{q}(a, t)=\tilde{p}(a, t)-\frac{1}{2}\left|\frac{\partial X(a, t)}{\partial t}\right|^{2} .
$$

We integrate (10) in time, fixing the label $a$ :

$$
\frac{\partial X^{j}(a, t)}{\partial t} \frac{\partial X^{j}(a, t)}{\partial a_{i}}=u_{(0)}^{i}(a)-\frac{\partial \tilde{n}(a, t)}{\partial a_{i}}
$$

where

$$
\tilde{n}(a, t)=\int_{0}^{t} \tilde{q}(a, s) d s
$$

and

$$
u_{(0)}(a)=\frac{\partial X(a, 0)}{\partial t}
$$

is the initial velocity. We consider

$$
A(x, t)=X^{-1}(x, t)
$$

the "back-to-labels" map, and note that it forms a vector of active scalars

$$
D_{t} A=\frac{\partial A}{\partial t}+u \cdot \nabla A=0 .
$$

Turning to (12), multiplying from the left by $\left[\left(\nabla_{a} X(a, t)\right)^{*}\right]^{-1}$, reading at $a=A(x, t)$, and denoting

$$
n(x, t)=\tilde{n}(A(x, t))
$$

we obtain the Weber formula

$$
u^{i}(x, t)=\left(u_{(0)}^{j}(A(x, t))\right) \frac{\partial A^{j}(x, t)}{\partial x_{i}}-\frac{\partial n(x, t)}{\partial x_{i}} .
$$


The equation (18) shows that the general Eulerian velocity can be written in a form:

$$
u=(\nabla A)^{*} B-\nabla n
$$

where $B=u_{(0)}(A(x, t))$ obeys

$$
D_{t} B=0 .
$$

Conversely, if one is given a pair $A=\left(A^{1}(x, t), \ldots, A^{M}(x, t)\right)$ and $B=$ $\left(B^{1}(x, t), \ldots, B^{M}(x, t)\right)$, if equations (16) and (20) hold and if $u$ is given by

$$
u(x, t)=\sum_{k=1}^{M} B^{k}(x, t) \nabla_{x} A^{k}(x, t)-\nabla_{x} n
$$

with some function $n$, then it follows that $u$ solves the Euler equations

$$
\frac{\partial u}{\partial t}+u \cdot \nabla u+\nabla \pi=0
$$

where

$$
\pi=D_{t} n+\frac{1}{2}|u|^{2} .
$$

Indeed, this is a consequence of the commutation relation

$$
D_{t} \nabla_{x} f=\nabla_{x} D_{t} f-\left(\nabla_{x} u\right)^{*} \nabla_{x} f
$$

that holds for any scalar function $f$. The kinematic commutation relation (22) follows from the chain rule, so it requires no assumption other than smoothness. Differentiating (21) and using (16), (20) it follows that

$$
\begin{aligned}
D_{t}(u)= & -\sum_{k=1}^{M}\left(\left(\nabla_{x} u\right)^{*} \nabla_{x} A^{k}\right) B^{k}-\nabla_{x}\left(D_{t} n\right)+\left(\nabla_{x} u\right)^{*} \nabla n \\
& -\nabla_{x}\left(D_{t} n\right)-\left(\nabla_{x} u\right)^{*}\left[\sum_{k=1}^{M}\left(\nabla_{x} A^{k}\right) B^{k}-\nabla_{x} n\right] \\
= & -\nabla_{x}\left(D_{t} n\right)-\left(\nabla_{x} u\right)^{*} u=-\nabla_{x}(\pi) .
\end{aligned}
$$

The previous calculations can be summarized as follows: A function $u(x, t)$ solves the incompressible Euler equations if and only if it can be represented in the form $u=u_{A}$ with

$$
u_{A}^{i}(x, t)=u_{(0)}^{m}(A(x, t)) \frac{\partial A^{m}(x, t)}{\partial x_{i}}-\frac{\partial n_{A}(x, t)}{\partial x_{i}}
$$

and

$$
\nabla \cdot u_{A}=0
$$


where $A(x, t)$ solves the equation

$$
\left(\partial_{t}+u_{A} \cdot \nabla\right) A=0
$$

with initial data

$$
A(x, 0)=x .
$$

The function $u_{(0)}$ represents the initial velocity and the function $n_{A}(x, t)$ is determined up to additive constants by the requirement of incompressibility, $\nabla \cdot u_{A}=0$ :

$$
\Delta n_{A}(x, t)=\frac{\partial}{\partial x_{i}}\left\{u_{(0)}^{m}(A(x, t)) \frac{\partial A^{m}(x, t)}{\partial x_{i}}\right\} .
$$

We will consider mainly periodic boundary conditions:

$$
A\left(x+L e_{j}, t\right)=A(x, t)+L e_{j} ; \quad n_{A}\left(x+L e_{j}, t\right)=n_{A}(x, t)
$$

with $e_{j}$ the standard basis in $\mathbb{R}^{3}$. In this case

$$
\delta_{A}(x, t):=x-A(x, t),
$$

$n_{A}(x, t)$, and $u_{A}(x, t)$ are periodic functions in each spatial direction. One may consider also the case of decay at infinity, requiring that $\delta_{A}$, $u_{A}$ and $n_{A}$ vanish sufficiently fast at infinity. The equation of state (23), (24) can be written as

$$
u_{A}=\mathbf{P}\left\{u_{(0)}^{m}(A(\cdot, t)) \nabla A^{m}(\cdot, t)\right\}=\mathbf{P}\left\{(\nabla A)^{*} u_{(0)}(A)\right\}
$$

where

$$
\mathbf{P}=\mathbb{I}-\nabla \Delta^{-1} \nabla
$$

is the Leray-Hodge projector on divergence-free functions.

The Eulerian pressure is determined, up to additive constants, from $n_{A}$ and $u_{A}$ by

$$
p(x, t)=\frac{\partial n_{A}(x, t)}{\partial t}+u_{A}(x, t) \cdot \nabla n_{A}(x, t)+\frac{1}{2}\left|u_{A}(x, t)\right|^{2} .
$$

The Jacobian of $A$ obeys

$$
\operatorname{det}(\nabla A(x, t))=1 .
$$

The vorticity

$$
\omega(x, t)=\omega_{A}(x, t)=\nabla \times u_{A}
$$

satisfies the Helmholtz equation

$$
D_{t} \omega=\omega \cdot \nabla u
$$


and is given by the Cauchy formula

$$
\omega(x, t)=\omega_{A}(x, t)=[\nabla A(x, t)]^{-1} \omega_{(0)}(A(x, t))
$$

where $\omega_{(0)}=\nabla \times u_{(0)}$ is the initial vorticity.

\section{Local Existence}

We have a local existence result in the Eulerian-Lagrangian formulation $([\mathbf{1 6}])$ :

Theorem 1. Let $u_{(0)}$ be a divergence-free $C^{1, \mu}$ periodic vector valued function of three variables. There exists a time interval $[0, T]$ and a unique $C\left([0, T] ; C^{1, \mu}\right)$ spatially periodic vector valued function $\delta(x, t)$ such that

$$
A(x, t)=x+\delta(x, t)
$$

solves the Eulerian-Lagrangian formulation of the Euler equations,

$$
\left\{\begin{array}{l}
\partial_{t} A+u \cdot \nabla A=0 \\
u=\mathbf{P}\left\{(\nabla A)^{*} v\right\} \\
\partial_{t} v+u \cdot \nabla v=0
\end{array}\right.
$$

with initial data $A(x, 0)=x, v(x, 0)=u_{(0)}$.

The same result holds if one replaces periodic boundary conditions with decay at infinity.

The idea of the proof follows below. Differentiating the equation (25) we obtain the equation obeyed by the gradients

$$
D_{t}\left(\frac{\partial A^{m}}{\partial x_{i}}\right)+\frac{\partial u_{A}^{j}}{\partial x_{i}} \frac{\partial A^{m}}{\partial x_{j}}=0 .
$$

We denote

$$
\mathbf{P}_{j l}=\delta_{j l}-\partial_{j} \Delta^{-1} \partial_{l}
$$

the matrix elements of the Leray-Hodge operator (with $\partial_{j}=\frac{\partial}{\partial x_{j}}$ ). Differentiating in the representation (28) and using the property

$$
\mathbf{P}_{j l} \frac{\partial f}{\partial x_{l}}=0
$$

we obtain

$$
\frac{\partial u_{A}^{j}}{\partial x_{i}}=\mathbf{P}_{j l}\left(\operatorname{Det}\left[\omega_{(0)}(A) ; \frac{\partial A}{\partial x_{i}} ; \frac{\partial A}{\partial x_{l}}\right]\right) .
$$

This relation shows that the gradient of velocity can be expressed without use of second order derivatives of $A$ and is the key to local existence: 
the equation (33) can be seen as a cubic quasi-local equation on characteristics. We consider the periodic case. We write $C^{j, \mu}, j=0,1$ to denote the Hölder spaces of real valued functions that are defined for all $x \in \mathbf{R}^{3}$ and are periodic with period $L$ in each direction. We denote by $\|f\|_{0, \mu}$ the $C^{0, \mu}$ norm:

$$
\|f\|_{0, \mu}=\sup _{x}|f(x)|+\sup _{x \neq y}\left\{|f(x)-f(y)|\left(\frac{L}{|x-y|}\right)^{\mu}\right\}
$$

and by $\|f\|_{1, \mu}$ the $C^{1, \mu}$ norm:

$$
\|f\|_{1, \mu}=\|f\|_{0, \mu}+L\|\nabla f\|_{0, \mu}
$$

where the notation $|\cdots|$ refers to modulus, Euclidean norm, and Euclidean norm for matrices, as appropriate.

We denote the map

$$
W[\delta, \phi](x, t)=\mathbb{P}\left\{(\mathbb{I}+\nabla \delta(x, t))^{*} \phi(x+\delta(x, t))\right\} .
$$

This map is linear in $\phi$ but nonlinear in $\delta$.

Proposition 1. The map $W[\delta, \phi]$ maps

$$
W:\left(C^{1, \mu}\right)^{3} \times\left(C^{1, \mu}\right)^{3} \rightarrow\left(C^{1, \mu}\right)^{3}
$$

continuously. There exist constants $C$ depending on $\mu$ alone so that

$$
\|W[\delta, \phi]\|_{0, \mu} \leq C\|\phi\|_{0, \mu}\left\{1+\|\nabla \delta\|_{0, \mu}\right\}^{2}
$$

and

$$
\|\nabla W[\delta, \phi]\|_{0, \mu} \leq C\|\nabla \times \phi\|_{0, \mu}\left\{1+\|\nabla \delta\|_{0, \mu}\right\}^{3}
$$

hold for any $\delta \in\left(C^{1, \mu}\right)^{3}, \phi \in\left(C^{1, \mu}\right)^{3}$.

We note that $W$ is made up from a number of operations. The first operation is the composition $\phi(x) \mapsto \phi(x+\delta(x))$. For a fixed $\delta \in\left(C^{1, \mu}\right)^{3}$ the map $x \mapsto x+\delta$ is Lipschitz. Composition with a Lipschitz change of variables maps $C^{0, \mu}$ into itself continuously. The joint continuity of $[\phi, \delta] \mapsto \phi(x+\delta)$ in $C^{1, \mu}$ follows naturally. The second operation is a sum of products of functions (a matrix applied to a vector). This is a continuous operation because the Hölder spaces $C^{j, \mu}, j=0,1$ we chose are Banach algebras. The third and last operation is the linear operator $\mathbf{P}$, which is bounded in Hölder spaces. We need to consider also derivatives of $W$. We use the formula (35) and note that the expression for the gradient is made of similar operations as above and apply the same kind of reasoning. 
We denote by $\Theta$ the map that associates to two continuous paths $t \mapsto \delta(\cdot, t)$ and $t \mapsto \phi(\cdot, t)$ a new path $t \mapsto \theta$; the path $t \mapsto \theta=\Theta[\delta, \phi]$ is obtained by solving the partial differential equation

$$
\frac{\partial \theta}{\partial t}+u \cdot \nabla \theta+u=0
$$

where

$$
u=W[\delta(\cdot, t), \phi(\cdot, t)],
$$

periodic boundary conditions are imposed on $\theta$ and zero initial data

$$
\theta(x, 0)=0
$$

are required. Let us consider the space

$$
\mathcal{P}_{T}=C\left([0, T],\left(C^{1, \mu}\right)^{3}\right)
$$

of continuous $\left(C^{1, \mu}\right)^{3}$-valued paths defined on a time interval $[0, T]$, endowed with the natural norm

$$
\|\theta\|_{1, \mathcal{P}}=\sup _{t}\|\theta(\cdot, t)\|_{1, \mu}
$$

We will consider also the weaker norm

$$
\|\theta\|_{0, \mathcal{P}}=\sup _{t}\|\theta(\cdot, t)\|_{0, \mu}
$$

$\Theta$ is nonlinear in both arguments.

Proposition 2. The map $\Theta[\delta, \phi]$ maps

$$
\Theta: \mathcal{P}_{T} \times \mathcal{P}_{T} \rightarrow \mathcal{P}_{T}
$$

and is continuous when the topology of the source space $\mathcal{P}_{T} \times \mathcal{P}_{T}$ is the natural product $C^{1, \mu}$ topology and the topology of the target space $\mathcal{P}_{T}$ is the weaker $C^{0, \mu}$ topology. Moreover, there exists a constant $C$ depending on $\mu$ alone so that

$$
\|\nabla \theta(\cdot, t)\|_{0, \mu} \leq\left(\int_{0}^{t}\|\nabla u(\cdot, s)\|_{0, \mu} d s\right)\left\{\exp \left\{C \int_{0}^{t}\|\nabla u(\cdot, s)\|_{0, \mu} d s\right\}\right\}
$$

holds for each $t \leq T$ with $u=W[\delta, \phi]$ and $\theta=\Theta[\delta, \phi]$.

Proposition 2 states that the map $\Theta$ is bounded in the strong $C^{1, \mu}$ topology. The proof follows naturally from use of the classical method of characteristics and ODE Gronwall type arguments. For fixed $\phi$, we take a small number $\epsilon>0$ and associate to it the set

$$
\mathcal{I} \subset \mathcal{P}_{T}
$$

defined by

$$
\mathcal{I}=\left\{\delta(x, t) ; \delta(x, 0)=0,\|\nabla \delta(\cdot, t)\|_{0, \mu} \leq \epsilon, \forall t \leq T\right\} .
$$


Combining the bounds in the two previous propositions one can choose, for fixed $\phi$, a $T$ small enough so that

$$
\delta \mapsto \Theta[\delta, \phi]=\mathcal{S}[\delta]
$$

maps

$$
\mathcal{S}: \mathcal{I} \rightarrow \mathcal{I}
$$

Inspecting the bounds it is clear that it is sufficient to require

$$
T\|\nabla \times \phi\|_{0, \mu} \leq c \epsilon
$$

with an appropriate $c$ depending on $\mu$ alone. We use the fact that $\delta_{j} \in \mathcal{I}$.

Proposition 3. Let $\phi \in\left(C^{1, \mu}\right)^{3}$ be fixed. There exists a constant depending on $\mu$ alone so that

$$
\left\|W\left[\delta_{1}, \phi\right]-W\left[\delta_{2}, \phi\right]\right\|_{0 \mu} \leq C\left\|\delta_{1}-\delta_{2}\right\|_{0, \mu}\|\phi\|_{1, \mu}
$$

holds for any $\delta_{1}, \delta_{2} \in C^{1, \mu}$ with $\left\|\delta_{j}\right\|_{1, \mu} \leq 1$.

One could use the condition $\delta_{j} \in C^{1, \mu}$ with $\left\|\delta_{j}\right\|_{1, \mu} \leq M$ but then $C$ would depend on $M$ also. Denoting

$$
\begin{gathered}
u=W\left[\delta_{1}, \phi\right]-W\left[\delta_{2}, \phi\right], \\
\delta=\delta_{1}-\delta_{2}, \\
\psi(x)=\frac{1}{2}\left(\phi\left(x+\delta_{1}(x)\right)+\phi\left(x+\delta_{2}(x)\right)\right), \\
v(x)=\phi\left(x+\delta_{1}(x)\right)-\phi\left(x+\delta_{2}(x)\right), \\
\gamma=\frac{1}{2}\left(\delta_{1}+\delta_{2}\right),
\end{gathered}
$$

we write

$$
u=u_{1}+u_{2}
$$

with

and

$$
u_{1}=\mathbf{P}\left\{(\nabla \delta)^{*} \psi\right\}
$$

The bound

$$
u_{2}=\mathbf{P}\left\{(\mathbf{I}+\nabla \gamma)^{*} v\right\}
$$

$$
\left\|u_{2}\right\|_{0, \mu} \leq C\|\delta\|_{0, \mu}\|\phi\|_{1, \mu}
$$

follows because $\phi$ is Lipschitz. The term $u_{1}$ requires special "integration by parts":

$$
u_{1}=-\mathbf{P}\left\{(\nabla \psi)^{*} \delta\right\},
$$

valid because of incompressibility. The matrix $\nabla \psi$ is bounded in $C^{0, \mu}$. We draw the attention to the fact that the presence of the $*$ (transpose) operation is essential for the "integration by parts" to be allowed. 
Leaving $\phi, \epsilon$ and $T$ fixed, the map $\mathcal{S}$ is Lipschitz in the weaker norm $C^{0, \mu}$ :

Proposition 4. There exists a constant $C$, depending on $\mu$ alone, such that, for every $\delta_{1}, \delta_{2} \in \mathcal{I}$, the Lipschitz bound

$$
\left\|\mathcal{S}\left[\delta_{1}\right]-\mathcal{S}\left[\delta_{1}\right]\right\|_{0, \mathcal{P}} \leq C\left\|\delta_{1}-\delta_{2}\right\|_{0, \mathcal{P}}
$$

holds.

We denote $\theta_{j}=\mathcal{S} \delta_{j}, u_{j}=W\left(\delta_{j}, \phi\right), u=u_{1}-u_{2}, \theta=\theta_{1}-\theta_{2}$ and write

$$
\frac{\partial \theta}{\partial t}+\frac{u_{1}+u_{2}}{2} \cdot \nabla \theta+u \cdot \nabla\left(\frac{\theta_{1}+\theta_{2}}{2}\right)+u=0 .
$$

We consider the characteristics $X(a, t)$ defined by

$$
\frac{d X}{d t}=\frac{u_{1}+u_{2}}{2}(X, t), \quad X((a, 0)=a
$$

and note that in view of Proposition 1 and the assumption $\delta_{j} \in \mathcal{I}$, the characteristics are well defined for $0 \leq t \leq T$, their inverse $A(x, t)=$ $X^{-1}(x, t)$ (the "back-to-labels" map) is defined too. Moreover,

$$
\sup _{t, a}\left|\frac{\partial X}{\partial a}\right| \leq C
$$

and

$$
\sup _{t, x}\left|\frac{\partial A}{\partial x}\right| \leq C
$$

holds with a constant $C$ depending on $\mu$ alone. Consider now the function

$$
F(x, t)=u \cdot \nabla\left(\frac{\theta_{1}+\theta_{2}}{2}\right)+u .
$$

Solving by the method of characteristics we obtain

$$
\theta(x, t)=-\int_{0}^{t} F(X(A(x, t), s), s) d s .
$$

Using Proposition 4 in conjunction with the bounds in Propositions 1 and 2 we see that $F(x, t)$ is bounded (uniformly in time) in $C^{0, \mu}$ :

$$
\sup _{t}\|F(\cdot, t)\|_{0, \mu} \leq C\|\phi\|_{1, \mu}\|\delta\|_{0, \mathcal{P}}
$$

Compositions with the uniformly Lipschitz $X$ and $A$ are harmless and we obtain the desired result

$$
\|\theta\|_{0, \mathcal{P}} \leq C\|\delta\|_{0, \mathcal{P}}
$$


The proof of Theorem 1 follows now using successive approximations. Starting with a first guess $\delta_{1} \in \mathcal{I}$ we define inductively

$$
\delta_{n+1}=\mathcal{S} \delta_{n} \in \mathcal{I}
$$

Proposition 3 can be used to show that the sequence $\delta_{n}$ converges rapidly in the $C^{0, \mu}$ topology to a limit $\delta$. Because $\mathcal{I}$ is convex it contains this weaker limit point, $\delta \in \mathcal{I}$. Because $\mathcal{S}$ has the weak Lipschitz property of Proposition 3 it follows that $\mathcal{S} \delta=\delta$. This actually means that $A=x+$ $\delta(x, t)$ solves the Eulerian-Lagrangian formulation of the Euler equations and that $u=W[\delta, \phi]$ solves the usual Eulerian formulation.

Now let us consider the case of decay at infinity. This case is instructive because it illuminates the difference between $\phi, u, W$ on the one hand and $x, \delta, \Theta$ on the other hand; the function spaces need to be modified in a natural fashion to accommodate this difference. The issue of decay at infinity is both a physical one - the total kinetic energy must be defined, and a mathematical one - $\mathbf{P}$ must be defined. But apart from this, the decay at infinity requirement does not hinder the proof in any respect.

Theorem 2. Let $u_{(0)}$ be a $C^{1, \mu}$ velocity that is square integrable

$$
\int\left|u_{(0)}(x)\right|^{2} d x<\infty
$$

and whose curl is integrable to some power $1<q<\infty$,

$$
\int\left|\nabla \times u_{(0)}(x)\right|^{q} d x<\infty .
$$

Then for $\epsilon$ sufficiently small there exists a time interval $[0, T]$ and a $C^{1, \mu}$ function $\delta(x, t)$ such that

$$
\sup _{t}\|\nabla \delta(\cdot, t)\|_{0, \mu} \leq \epsilon
$$

and such that $x+\delta(x, t)$ solves the Eulerian-Lagrangian formulation of the Euler equation. The velocity corresponding to this solution belongs to $C^{1, \mu}$, is square integrable and the vorticity is integrable to power $q$.

The proof follows the same lines as above. Because $u_{(0)}$ enters linearly in the expression for $W$ and because we control $\nabla \delta$ uniformly, issues of decay at infinity of do not arise. In other words, the function space for velocities does not need to be a Banach algebra, rather a module over the Banach algebra of the $\delta$ variables, which need not decay at infinity. 


\section{The Blow Up Issue for Euler Equations}

The blow up problem for the Euler equations is: given smooth initial data with finite energy, does any singularity form in finite time? This problem is open. If the energy is not finite, then stagnation point singularities do appear in finite time $([\mathbf{5 2}])$ but such infinite energy singularities appear even in two dimensions $([\mathbf{9}])$, while, as is very well known, finite energy solutions remain smooth in two dimensions. Different infinite-energy, three dimensional blow up does occur $([\mathbf{4 6}],[\mathbf{1 5}])$. The blow up is caused by a singularity in the gradient of velocity.

The equation obeyed by the matrix $G$ of gradients of velocities of solutions of the Euler equation is

$$
D_{t} G+[\mathbb{I}+(R \otimes R) \mathrm{T} r] G^{2}=0 .
$$

$R=\nabla(-\Delta)^{-\frac{1}{2}}$ are Riesz transforms and $(R \otimes R) \operatorname{Tr} G^{2}$ is the Hessian of the pressure, $\partial_{i} \partial_{j} p$. This quadratic nonlocal equation blows up in finite time if $D_{t}$ is replaced by $\partial_{t}([\mathbf{1 2}])$. The anti-symmetric part of this equation is local (30). The well-known Beale-Kato-Majda criterion ([2]) states that, if the initial data are smooth, and if

$$
\int_{0}^{T}\|\omega(\cdot, t)\|_{L^{\infty}(d x)} d t<\infty
$$

then the solution is smooth on $[0, T]$. Therefore the vorticity magnitude controls blow up. The magnitude evolves according to the stretching equation

$$
D_{t}(|\omega|)=\alpha|\omega| .
$$

The stretching factor $\alpha$ is related to the vorticity magnitude through a principal value singular integral $([\mathbf{1 4}])$ :

$$
\alpha(x, t)=P . V . \int D(\hat{y}, \xi(x, t), \xi(x+y, t))|\omega(x+y, t)| \frac{d y}{|y|^{3}} .
$$

Here $\hat{y}$ is the unit vector in the direction of $y, \xi(x, t)=\frac{\omega}{|\omega|}$ is the unit vector tangent to the vortex line passing through $x$ at time $t$ and $D$ is a certain geometric factor. The geometric factor is a smooth function of three unit vectors, has zero average on the unit sphere, $\int D d S(\hat{y})=0$ and vanishes pointwise when $\xi(x, t)= \pm \xi(x+y, t)$. Because $\alpha$ has the same order of magnitude as $|\omega|$, dimensional reasoning suggests blow up of the type one encounters in the ordinary differential equation $\frac{d m}{d t}=m^{2}$,

$$
\sup _{x}|\omega(x, t)| \sim \frac{1}{T-t} .
$$


But if the vorticity direction $\xi$ is smooth then a geometric depletion of $\alpha$ occurs; that means that $\alpha$ is of the order of magnitude of velocity times the magnitude of the spatial gradient of $\xi$ (an inverse length scale, assumed to be finite). The two dimensional Euler equations correspond to the case $\xi=(0,0,1)$ and $\alpha=0$ identically. If

$$
\int_{0}^{T}\|\alpha(\cdot, t)\|_{L^{\infty}} d t<\infty
$$

then no blow up can occur. The geometric depletion of nonlinearity has been investigated theoretically and numerically for the three dimensional Euler and Navier-Stokes equations and for the two dimensional surface quasi-geostrophic equation $([\mathbf{3}],[\mathbf{5}],[\mathbf{6}],[\mathbf{1 4}],[\mathbf{2 0}],[\mathbf{2 1}],[\mathbf{2 3}],[\mathbf{2 4}],[\mathbf{2 6}]$, $[27],[28],[30],[29],[32],[31],[48],[40],[47])$

We discuss the blow up issues in the context of a two-dimensional example, the ideal Boussinesq system. The reason for this is that two spatial dimensional systems are somewhat easier to visualize and compute, and the Boussinesq system is a physical model that presents some of the challenges associated with the $3 \mathrm{D}$ blow up probems.

$$
\left\{\begin{array}{l}
\left(\partial_{t}+u \cdot \nabla\right) u+\nabla p=g \theta e_{2} \\
\left(\partial_{t}+u \cdot \nabla\right) \theta=0 \\
\nabla \cdot u=0
\end{array}\right.
$$

The unit vector $e_{2}$ points in the direction opposite to gravity and the constant $g$ has units of acceleration. The vorticity $\omega=\nabla^{\perp} \cdot u$ obeys

$$
\left(\partial_{t}+u \cdot \nabla\right) \omega=g \partial_{1} \theta
$$

The vector $\nabla^{\perp} \theta$ obeys the equation

$$
\left(\partial_{t}+u \cdot \nabla\right) \nabla^{\perp} \theta=(\nabla u) \nabla^{\perp} \theta .
$$

Let us consider the inverse Lagrangian map $A(x, t)$ that obeys

$$
\left(\partial_{t}+u \cdot \nabla\right) A=0
$$

with initial data $A(x, 0)=x$. The direct Lagrangian map is denoted $X(a, t)$. We denote by

$$
(f \circ X)(a, t)=f(X(a, t), t)
$$

the composition with the direct Lagrangian map. The direct map obeys $X(A(x, t), t)=x$ and

$$
\partial_{t} X=u \circ X
$$


We denote by $\{f, g\}=\left(\partial_{1} f\right)\left(\partial_{2} g\right)-\left(\partial_{2} f\right)\left(\partial_{1} g\right)=\left(\nabla^{\perp} f\right) \cdot(\nabla g)$ the usual Poisson bracket. Using incompressibility one verifies that

$$
\{f, g\} \circ X=\{f \circ X, g \circ X\} .
$$

Clearly

$$
\partial_{t}(f \circ X)=\left(\left(\partial_{t}+u \cdot \nabla\right) f\right) \circ X
$$

and

$$
\theta \circ X=\theta_{0}
$$

where $\theta_{0}$ is the initial datum for $\theta$. Derivatives obey

(49) $\quad\left(\partial_{1} f\right) \circ X=\left\{f \circ X, X_{2}\right\}, \quad\left(\partial_{2} f\right) \circ X=-\left\{f \circ X, X_{1}\right\}$.

Using (47), (48), (49))it follows from (43) that

$$
\partial_{t}(\omega \circ X)=g\left\{\theta_{0}, X_{2}\right\}
$$

and, denoting by $\left(\nabla^{\perp} \theta\right) \circ X$ by $\tau \circ X$, it follows from (44) that

$$
\partial_{t}(\tau \circ X)=\left\{\theta_{0}, u \circ X\right\} .
$$

Integrating this we get

$$
\tau \circ X=\left\{\theta_{0}, X\right\},
$$

which is just the Lagrangian counterpart of the Eulerian-Lagrangian

$$
\nabla^{\perp} \theta(x, t)=\left(\frac{\partial \theta_{0}}{\partial a_{l}}(A(x, t))\right) \nabla^{\perp} A_{l}(x, t)
$$

which is an analogue of the Cauchy formula (31).

Proposition 5. Assume that the initial data $u_{(0)}$ and $\theta_{(0)}$ of (42) belong to $W^{2, q}\left(\mathbb{R}^{2}\right)$ for $q>2$. Assume that $u$ is locally Lipschitz on the time interval $[0, T]$ and that

$$
\int_{0}^{T}\|\nabla u\|_{L^{\infty}\left(\mathbb{R}^{2}\right)} d t<\infty .
$$

Then

$$
\int_{0}^{T}\|\nabla A\|_{L^{\infty}\left(\mathbb{R}^{2}\right)} d t<\infty
$$

holds and the solution $(u, \theta)$ belongs to $L^{\infty}\left([0, T], W^{2, q}\left(\mathbb{R}^{2}\right)\right)$. 
Indeed, differentiating (45) we obtain (55) immediately from (54). In view of (53) it follows that

$$
\int_{0}^{T}\|\nabla \theta\|_{L^{\infty}\left(\mathbb{R}^{2}\right)} d t \leq G<\infty .
$$

Incompressibility and two dimensionality imply the fact that $\nabla_{a} X$ is a linear combination of $\left(\nabla_{x} A\right) \circ X$. This in turn implies that

$$
\int_{0}^{T}\left\|\nabla_{a} X\right\|_{L^{\infty}\left(\mathbb{R}^{2}\right)} d t<\infty
$$

and, in view of (50), we deduce

$$
\sup _{t \leq T}\|\omega\|_{L^{r}\left(\mathbb{R}^{2}\right)}<\infty
$$

for $r \geq q$.

Differentiating (44) and using $\|\nabla \nabla u\|_{L^{q}\left(\mathbb{R}^{2}\right)} \leq C\|\nabla \omega\|_{L^{q}\left(\mathbb{R}^{2}\right)}$ we obtain

(57) $\frac{d}{d t}\|\nabla \nabla \theta\|_{L^{q}\left(\mathbb{R}^{2}\right)}$

$$
\leq C\|\nabla u\|_{L^{\infty}\left(\mathbb{R}^{2}\right)}\|\nabla \nabla \theta\|_{L^{q}\left(\mathbb{R}^{2}\right)}+C\|\nabla \theta\|_{L^{\infty}\left(\mathbb{R}^{2}\right)}\|\nabla \omega\|_{L^{q}\left(\mathbb{R}^{2}\right)}
$$

and differentiating (43) we have

$$
\frac{d}{d t}\|\nabla \omega\|_{L^{q}\left(\mathbb{R}^{2}\right)} \leq C\|\nabla u\|_{L^{\infty}\left(\mathbb{R}^{2}\right)}\|\nabla \omega\|_{L^{q}\left(\mathbb{R}^{2}\right)}+g\|\nabla \nabla \theta\|_{L^{q}\left(\mathbb{R}^{2}\right)} .
$$

Adding these inequalities and using (54) and (56) we finish the proof. Let us consider the stream function $\psi$ is given by

$$
\psi(x, t)=\frac{1}{2 \pi} \int_{\mathbb{R}^{2}} \log (|x-y|) \omega(y, t) d y .
$$

After a brief calculation using $u=\nabla^{\perp} \psi$ we obtain

(60) $\frac{\partial u_{i}}{\partial x_{j}}(x, t)=\frac{1}{4 \pi} \epsilon_{j i} \omega(x, t)+\frac{1}{2 \pi} P . V . \int_{\mathbb{R}^{2}} \frac{\epsilon_{j i}-2\left(\hat{z}_{j}\right)\left(\hat{z}_{i}^{\perp}\right)}{|z|^{2}} \omega(x-z, t) d z$

where $\epsilon_{j i}$ is the signature of the permutation $(1,2) \mapsto(j, i)$ and $\hat{z}=\frac{z}{|z|}$ is the unit vector in the direction $z \in \mathbb{R}^{2}$. Using this representation, we can prove 
Lemma 1. Let $u=\nabla^{\perp} \psi$ be a divergence-free vector obtained from a bounded and integrable vorticity function $\omega$ via a stream function $\psi(59)$. For any $q>2$, and $p<\infty$ there exists a constant $C$ such that

(61) $\quad\|\nabla u\|_{L^{\infty}\left(\mathbb{R}^{2}\right)}$

$$
\leq C\|\omega\|_{L^{\infty}}\left\{1+\log _{+}\left[\|\omega\|_{L^{p}\left(\mathbb{R}^{2}\right)}^{\frac{p(q-2)}{2 q}}\|\omega\|_{L^{\infty}\left(\mathbb{R}^{2}\right)}^{-\frac{(2+p) q-2 p}{2 q}}\|\nabla \omega\|_{L^{q}\left(\mathbb{R}^{2}\right)}\right]\right\} .
$$

The proof of this lemma is done by splitting the integral in $(60)$ in three pieces, an inner region, $|z| \leq \delta$, an intermediate region $\delta<|z|<L$ and an outer region $|z| \geq L$. One may choose first $L$ as the length scale defined with $\|\omega\|_{L^{\infty}\left(\mathbb{R}^{2}\right)}$ and $\|\omega\|_{L^{p}\left(\mathbb{R}^{2}\right)}$,

$$
L^{\frac{2}{p}}=\frac{\|\omega\|_{L^{p}\left(\mathbb{R}^{2}\right)}}{\|\omega\|_{L^{\infty}\left(\mathbb{R}^{2}\right)}} .
$$

The outer piece is bounded by $C L^{-\frac{2}{p}}\|\omega\|_{L^{p}\left(\mathbb{R}^{2}\right)}=C\|\omega\|_{L^{\infty}\left(\mathbb{R}^{2}\right)}$ and the intermediate region by $C\|\omega\|_{L^{\infty}\left(\mathbb{R}^{2}\right)} \log _{+}\left(\frac{L}{\delta}\right)$. The inner region can be bounded using the structure of the kernel by $C \delta^{1-\frac{2}{q}}\|\nabla \omega\|_{L^{q}\left(\mathbb{R}^{2}\right)}$. The proof of the lemma ends by choosing $\delta$ so that the inner piece is also bounded by $C\|\omega\|_{L^{\infty}\left(\mathbb{R}^{2}\right)}$.

Now let us assume that we know only the bound (56). Then, using (43) together with (44) we have

$$
\begin{aligned}
& \frac{d}{d t}\left\{\|\omega\|_{L^{r}\left(\mathbb{R}^{2}\right)}+\|\nabla \theta\|_{L^{r}\left(\mathbb{R}^{2}\right)}\right\} \\
& \quad \leq\left(\|\nabla \theta\|_{L^{\infty}\left(\mathbb{R}^{2}\right)}+g\right)\left\{\|\omega\|_{L^{r}\left(\mathbb{R}^{2}\right)}+\|\nabla \theta\|_{L^{r}\left(\mathbb{R}^{2}\right)}\right\}
\end{aligned}
$$

and consequently

$$
\sup _{t \leq T}\left\{\|\omega\|_{L^{r}\left(\mathbb{R}^{2}\right)}+\|\nabla \theta\|_{L^{r}\left(\mathbb{R}^{2}\right)}\right\} \leq \Omega_{r}<\infty
$$

for $r \geq q$, with $\Omega_{r}$ depending on $G$ and initial data. Adding (57) and (58) we have

$$
\frac{d Y}{d t} \leq C\left(\|\nabla \theta\|_{L^{\infty}\left(\mathbb{R}^{2}\right)}+\|\nabla u\|_{L^{\infty}\left(\mathbb{R}^{2}\right)}+g\right) Y(t)
$$

for

$$
Y(t)=\|\nabla \nabla \theta\|_{L^{q}\left(\mathbb{R}^{2}\right)}+\|\nabla \omega\|_{L^{q}\left(\mathbb{R}^{2}\right)} .
$$

In view of (62) and (61) it follows from the Gronwall inequality that

$$
\sup _{t \leq T}\left[\|\nabla \nabla \theta\|_{L^{q}\left(\mathbb{R}^{2}\right)}+\|\nabla \omega\|_{L^{q}\left(\mathbb{R}^{2}\right)}\right] \leq C
$$

holds with $C$ depending only on initial data, $T$ and $G$. Thus 
Proposition 6. Let $T>0$, and let $\left(u_{(0)}, \theta_{0}\right) \in W^{2, q}\left(\mathbb{R}^{2}\right)$ with $q>2$ be initial data for (42). Assume that the solution $(u, \theta)$ is smooth on the interval $[0, T)$. If the solution blows up at $T$ then

$$
\int_{0}^{T}\|\nabla \theta\|_{L^{\infty}\left(\mathbb{R}^{2}\right)} d t=\infty .
$$

Conversely, if the integral above is finite, then $(u, \theta)$ can be extended uniquely beyond $T$,

$$
(u, \theta) \in L^{\infty}\left(\left[0, T_{1}\right], W^{2, q}\left(\mathbb{R}^{2}\right)\right)
$$

with $T_{1}>T$.

This shows that the gradient of $\theta$ controls blow up in the ideal Boussinesq equations $([\mathbf{7}],[\mathbf{3 3}])$. The magnitude

$$
m(x, t)=\left|\nabla^{\perp} \theta(x, t)\right|
$$

obeys

$$
\left(\partial_{t}+u \cdot \nabla\right) m(x, t)=\alpha(x, t) m(x, t)
$$

with

$$
\begin{gathered}
\alpha(x, t)=S(x, t) \xi(x, t) \cdot \xi(x, t), \\
S=\left((\nabla u)+(\nabla u)^{*}\right) / 2, \quad \xi=\frac{\nabla^{\perp} \theta}{\left|\nabla^{\perp} \theta\right|} .
\end{gathered}
$$

Using (60) we have

$$
S(x, t)=-\frac{1}{2 \pi} P . V . \int \frac{\left(\hat{z}_{j}\right)\left(\hat{z}_{i}^{\perp}\right)+\left(\hat{z}_{i}\right)\left(\hat{z}_{j}^{\perp}\right)}{|z|^{2}} \omega(x-z, t) d z,
$$

and consequently

$$
\alpha(x, t)=\frac{1}{\pi} P . V \cdot \int \frac{(\hat{z} \cdot \xi(x, t))\left(\hat{z} \cdot \xi^{\perp}(x, t)\right)}{|z|^{2}} \omega(x-z, t) d z .
$$

At present we do not know how to prove that blow up does not occur, nor that it does. The mathematical analysis can shed some light on numerical studies, however. Unpublished numerical studies by the group of Professor S. Toh in Kyoto, showed that a putative blow up occurs at time $t_{*}$ and that the nature of the blow up is the following. There exists a length scale $\delta(t)$ that converges to zero, $\lim _{t \rightarrow t_{*}} \delta(t)=0$ so that the gradient of $\theta$ changes significantly only in a strip of width $\delta(t)$ along a line corresponding to a particular value of $\theta$. The line itself does not change much in time. 
To investigate this mathematically we take, without loss of generality, coordinates so that this line is the $x_{1}$ axis. The computed velocity is approximately a shear, that is, that it changes significantly across this line, but not away from it, and that at the putative blow-up time the vorticity concentrates along the same line. This shear formation would be described to leading order by $u\left(x_{1}, x_{2}, t\right)=U\left(\frac{x_{2}}{\delta}, t\right) \widehat{x_{1}}$, with $U$ analytic, but non-constant. The vorticity generated from such a velocity would be $\omega\left(x_{1}, x_{2}, t\right)=-\frac{1}{\delta} U_{Y}\left(\frac{x_{2}}{\delta}, t\right)$. This vorticity does not contribute to $\alpha(x)$. More precisely if the putative blow up point is $x=\left(x_{1}, x_{2}\right)=(0,0)$ then the direction $\xi(0, t)$ is $\widehat{x_{1}}$ and the integral representing $\alpha(0, t)$ is, to leading order, equal to

$$
\alpha(0,0, t) \sim \frac{1}{\pi} P . V . \int_{0}^{\infty} \int_{0}^{2 \pi} \sin \phi \cos \phi U_{Y}\left(-r \frac{1}{\delta} \sin \phi\right) \frac{1}{\delta r} d \phi d r=0 .
$$

The integral vanishes because the integrand is a derivative of an analytic function of $\sin \phi$. So the only contributions to the growth of $\nabla \theta$ come from the subleading order terms. These will be bounded, if for instance $u\left(x_{1}, x_{2}, t\right)=U\left(\delta x_{1}, \frac{1}{\delta} x_{2}, t\right)$, i.e. if the variation of the velocity along the putative line of blow up is slow. Only if this variation is rapid can one sustain a blow up. This means however that the vortex sheet would have to crumple, on scales that vanish together with $\delta$. The crumpling was observed numerically at the edges of the sheet.

\section{Navier-Stokes Equations}

The Navier-Stokes Equations $([\mathbf{2 2}],[\mathbf{4 2}])$ are

$$
\begin{aligned}
D_{\nu} u+\nabla p & =0, \\
\nabla \cdot u & =0 .
\end{aligned}
$$

The operator $D_{\nu}$

$$
D_{\nu}=D_{\nu}(u, \nabla)=\partial_{t}+u \cdot \nabla-\nu \Delta
$$

describes advection with velocity $u$ and diffusion with kinematic viscosity $\nu>0$. When $\nu=0$ we recover the Euler equations (1), and $D_{\nu \mid \nu=0}=D_{t}$. In the smooth regime this an obvious result; in nonsmooth regimes the problem is nontrivial ([25]) and in the presence of boundaries, the limit is not completely understood. The vorticity $\omega=\nabla \times u$ obeys an equation similar to (30):

$$
D_{\nu} \omega=\omega \cdot \nabla u \text {. }
$$


The Eulerian-Lagrangian representation of the Euler equations (32) has a viscous counterpart $([\mathbf{1 7}],[\mathbf{1 8}])$ :

$$
\left\{\begin{array}{l}
D_{\nu} A=0, \\
D_{\nu} v=2 \nu C \nabla v, \\
u=\mathbb{P}\left((\nabla A)^{*} v\right) .
\end{array}\right.
$$

The right hand side of (70) is given terms of the connection coefficients

$$
C_{k ; i}^{m}=\left((\nabla A)^{-1}\right)_{j i}\left(\partial_{j} \partial_{k} A^{m}\right) .
$$

The detailed form of virtual velocity equation in (70) is

$$
D_{\nu} v_{i}=2 \nu C_{k ; i}^{m} \partial_{k} v_{m} .
$$

In addition to being locally well posed, the Eulerian-Lagrangian viscous equations are capable of describing vortex reconnection. We associate to the virtual velocity $v$ the Eulerian-Lagrangian curl of $v$

$$
\zeta=\nabla^{A} \times v
$$

where

$$
\nabla_{i}^{A}=\left((\nabla A)^{-1}\right)_{j i} \partial_{j}
$$

is the pull back of the Eulerian gradient. The vorticity equation representation in Eulerian-Lagrangian form is

$$
\left\{\begin{array}{l}
D_{\nu} A=0, \\
D_{\nu} \zeta^{q}=2 \nu G_{p}^{q k} \partial_{k} \zeta^{p}+\nu T_{p}^{q} \zeta^{p}, \\
u=\nabla \times(-\Delta)^{-1}(\mathcal{C}[\nabla A, \zeta]) .
\end{array}\right.
$$

The Cauchy transformation

$$
\mathcal{C}[\nabla A, \zeta]=(\operatorname{det}(\nabla A))(\nabla A)^{-1} \zeta
$$

is the same as the one used in the Euler equations, except that $\operatorname{det}(\nabla A)$ need not be identically equal to 1 . The specific form of the two terms on the right hand side of the Cauchy invariant's evolution are

$$
G_{p}^{q k}=\delta_{p}^{q} C_{k ; m}^{m}-C_{k ; p}^{q}
$$

and

$$
T_{p}^{q}=\epsilon_{q j i} \epsilon_{r m p} C_{k ; i}^{m} C_{k ; j}^{r} .
$$

The pair $(A, v)$ formed by the diffusive inverse Lagrangian map and the virtual velocity are akin to charts in a manifold. They are a convenient representation of the dynamics of $u$ for some time. When the representation becomes inconvenient, then one has to change the chart. This may (and will) happen if $\nabla A$ becomes non-invertible. Likewise, the 
pair $(A, \zeta)$ formed with the "back-to-labels" map $A$ and the diffusive Cauchy invariant $\zeta$ are convenient charts. Because the fluid variables $u$ or $\omega$ are represented as products of elements in the chart, it is possible for the chart to become singular without the fluid becoming singular. The regularity of the fluid is not equivalent to the regularity of a single chart, but rather to the existence of smooth, compatible charts. In order to quantify this statement we used the terminology of "group expansion" for the procedure of resetting. More precisely, the group expansion for (70) is defined as follows. Given a time interval $[0, T]$ we consider resetting times

$$
0=t_{0}<t_{1}<\cdots<t_{n} \cdots \leq T .
$$

On each interval $\left[t_{i}, t_{i+1}\right], i=0, \ldots$ we solve the system $(70)$ :

$$
\left\{\begin{array}{l}
D_{\nu}(u, \nabla) A=0 \\
D_{\nu}(u, \nabla) v=2 \nu C \nabla v \\
u=\mathbf{P}\left((\nabla A)^{*} v\right)
\end{array}\right.
$$

with resetting conditions

$$
\left\{\begin{array}{l}
A\left(x, t_{i}\right)=x, \\
v\left(x, t_{i}+0\right)=\left((\nabla A)^{*} v\right)\left(x, t_{i}-0\right) .
\end{array}\right.
$$

The resetting occurs if $\nabla \ell=(\nabla A)-\mathbf{I}$ reaches a preassigned value $\epsilon$ in an analytic norm, where $\ell:=A-x$. In fact, we fix $\lambda>0$ and require that for all $i \geq 1$ and all $t \in\left[t_{i}, t_{i+1}\right)$ the inequality

$$
\int e^{\lambda|k|}|\widehat{\ell}(k)| d k<\epsilon<1
$$

is satisfied. If there exists $N$ such that $T=\sum_{i=0}^{N}\left(t_{i+1}-t_{i}\right)$ then we say that the group expansion converges on $[0, T]$. A group expansion of (72) is defined similarly. The resetting conditions are

$$
\left\{\begin{array}{l}
A\left(x, t_{i}\right)=x, \\
\left.\zeta\left(x, t_{i}+0\right)=\mathcal{C}[(\nabla A))\left(x, t_{i}-0\right), \zeta\left(x, t_{i}-0\right)\right] .
\end{array}\right.
$$

The analytic resetting criterion is the same. The first interval of time $\left[0, t_{1}\right)$ is special. The initial value for $v$ is $u_{0}$ (the initial datum for the Navier-Stokes solution), and the initial value for $\zeta$ is $\omega_{0}$, the corresponding vorticity. The local time existence is used to guarantee invertibility of the matrix $\nabla A$ on $\left[0, t_{1}\right)$ and Gevrey regularity $([\mathbf{3 8}])$ to pass from moderately smooth initial data to Gevrey class regular solutions. Note that the resetting conditions are designed precisely so that 
both $u$ and $\omega$ are time continuous. The following result was proved in $[\mathbf{1 8}]$.

Theorem 3. Let $u_{0} \in H^{1}\left(\mathbf{R}^{3}\right)$ be divergence-free. Let $T>0$. Assume that the solution of the Navier-Stokes equations with initial datum $u_{0}$ obeys $\sup _{0 \leq t \leq T}\|\omega(\cdot, t)\|_{L^{2}(d x)}<\infty$. Then there exists $\lambda>0$ so that, for any $\epsilon>0$, there exists $\tau>0$ such that the group expansions for both (70) and (72) converge on $[0, T]$ and the resetting intervals can be chosen to have any length up to $\tau, t_{i+1}-t_{i} \in[0, \tau]$.

Conversely, if one group expansion converges, then so does the other, using the same resetting times. The Weber and Cauchy formulas apply and reconstruct the solution of the Navier-Stokes equation. The enstrophy is bounded $\sup _{0 \leq t \leq T}\|\omega(\cdot, t)\|_{L^{2}(d x)}<\infty$, and the Navier-Stokes solution is smooth.

The Navier-Stokes equations require frequent resettings. There is a deep connection between these resetting times and vortex reconnection ([45]). In the Euler equation, as long as the solution is smooth, the Cauchy invariant obeys $\zeta(x, t)=\omega_{(0)}(A(x, t))$ with $\omega_{(0)}=\omega_{0}$, the initial vorticity. The topology of vortex lines is frozen in time. In the Navier-Stokes system the topology changes. This is the phenomenon of vortex reconnection. Vortex reconnection is a dynamical dissipative process. The solutions of the Navier-Stokes equations obey a space time average bound $([\mathbf{1 3}])$

$$
\int_{0}^{T} \int_{\mathbb{R}^{3}}|\omega(x, t)|\left|\nabla_{x}\left(\frac{\omega(x, t)}{|\omega(x, t)|}\right)\right|^{2} d x d t \leq \frac{1}{2} \nu^{-2} \int_{\mathbf{R}^{3}}\left|u_{0}(x, t)\right|^{2} d x .
$$

This bound is consistent with the numerically observed fact that the region of high vorticity is made up of relatively straight vortex filaments separated by distances of the order of the Kolmogorov length. The processes by which these configurations are obtained and sustained are vortex stretching and vortex reconnection. When vortex lines are locally aligned, a geometric depletion of nonlinearity occurs, and the local production of enstrophy drops. Indeed, the Navier-Stokes equations have global smooth solutions if the vorticity direction field $\frac{\omega}{|\omega|}$ is Lipschitz continuous ([20]) in regions of high vorticity. Vortex reconnection is a manifestation of a regularizing mechanism.

\section{The Blow Up Issue for Navier-Stokes Equations}

If the solution of the Euler equations with initial data $u_{(0)}$ is smooth on a time interval $[0, T]$ then the solutions of the Navier-Stokes equations 
with the same initial data are smooth on the same time interval, if $\nu>0$ is small enough $([\mathbf{1 2}])$. The Navier-Stokes blow up is not a gradient blow up: if

$$
\int_{0}^{T}\|u\|_{L^{\infty}(d x)}^{2} d t<\infty
$$

then the solution is smooth on $[0, T]([\mathbf{5 1}])$. The known result in this context is

$$
\int_{0}^{T}\|u\|_{L^{\infty}(d x)} d t<\infty .
$$

This result ([17]) follows using the ideas of $[\mathbf{3 7}]$. The sufficient conditions for regularity involving gradients are obtained easily. Multiplying the vorticity equation (69) by $\omega$, integrationg and using Morrey-Sobolev inequalities and Holder inequalities we arrive at

$$
\frac{d}{d t}\|\omega\|_{L^{2}(d x)}^{2}+\nu\|\nabla \omega\|_{L^{2}(d x)}^{2} \leq C \nu^{-3}\|\omega\|_{L^{2}(d x)}^{6} .
$$

If

$$
\int_{0}^{T}\|\omega\|_{L^{2}(d x)}^{4} d t<\infty
$$

it follows thus that $u$ is a strong solution $\left(u \in L^{\infty}\left([0, T], W^{1,2}\right) \cap L^{2}([0, T] \cap\right.$ $\left.W^{2,2}\right)$ ) and therefore smooth $([\mathbf{2 2}])$. The geometric depletion of nonlinearity can be used in conjunction with this, and one can formulate conditions sufficient for regularity in terms of only the direction field associated to the vorticity $([\mathbf{2 0}])$. Conditions sufficient for regularity in terms of only the pressure are given in $[49]$. The completion of the range of conditions in terms of only the velocity is given in [35].

The global regularity of solutions can be pursued in the context of the Eulerian-Lagrangian formulations (70). Here we give a condition in terms of only the back-to-labels map. We pick a fixed a small number $\epsilon>$ 0 and require on each interval of time $I=\left[t_{i}, t_{i+1}\right]$

$$
\sup _{t \in I}\|\nabla \ell\|_{L^{\infty}(d x)} \leq \epsilon
$$


The equation obeyed by $\ell=A-\mathbb{I}$ is

$$
D_{\nu} \ell+u=0
$$

with $\ell\left(x, t_{i}\right)=0$. Therefore,

$$
\nabla \ell(t)=-\int_{t_{i}}^{t} e^{\nu(t-s) \Delta} \nabla\{(\mathbb{I}+\nabla \ell(s))\} u(s) d s .
$$

Denoting $g(s)=\|\nabla \ell(\cdot, s)\|_{L^{\infty}(d x)}$, and using well known properties of the heat kernel, we have

$$
g(t) \leq C \int_{t_{i}}^{t}(\nu(t-s))^{-\frac{p+3}{2 p}}(1+g(s))\|u(s)\|_{L^{p}(d x)} d s
$$

for $p>3$. Using the Weber formula (70) we have

$$
\|u(s)\|_{L^{p}(d x)} \leq C(1+g(s))\|v(s)\|_{L^{p}(d x)}
$$

and thus $\widetilde{g}(t)=\sup _{t_{i} \leq s \leq t} g(s)$ obeys

$$
\frac{\widetilde{g}(t)}{1+\widetilde{g}(t)} \leq C \nu^{-\frac{p+3}{2 p}} \tau^{\left(\frac{p-3}{2 p}-\frac{1}{q}\right)}\left\{\int_{t_{i}}^{t}\|v(s)\|_{L^{p}(d x)}^{q} d s\right\}^{\frac{1}{q}}
$$

if $q>\frac{2 p}{p-3}$, and $\tau=t_{i+1}-t_{i}$. Using $\epsilon \leq 1$ we deduce that, if

$$
\left(\int_{I}\|v(s)\|_{L^{p}(d x)}^{q} d s\right)^{\frac{1}{q}} \leq C_{p q}
$$

holds then (76) holds if we choose $\tau=t_{i+1}-t_{i}$ small enough. In particular, if

$$
V_{p}(I)=\sup _{t \in I}\|v(t)\|_{L^{p}(d x)}
$$

is finite, with $p>3$ then

$$
C \tau^{\frac{p-3}{2 p}} \nu^{-\frac{p+3}{2 p}} V_{p}(I) \leq \epsilon
$$

implies (76). 
The semigroup method is suitable for $\ell$ because $\ell$ is small, but it is not suitable for $v$. The fact that an equation of the type $D_{\nu} v=0$ does not increase $L^{p}$ norms for large smooth $u$ is not easily seen using the semigroup method, but it is transparent using the energy method. We take the $v$ equation of (70), multiply by $v|v|^{p-2}$, integrate, and use the divergence-free condition:

$$
\frac{1}{p} \frac{d}{d t} \int|v|^{p} d x+\nu \int|\nabla v|^{2}|v|^{p-2} d x \leq C \nu \int|\nabla v \| v|^{p-1}|C(x, t)| d x .
$$

A Schwartz inequality brings us to

$$
\frac{1}{p} \frac{d}{d t} \int|v|^{p} d x+\nu \int|\nabla v|^{2}|v|^{p-2} d x \leq C \nu \int|v|^{p}|C(x, t)|^{2} d x,
$$

and therefore, using Hölder

$$
\begin{aligned}
& \frac{1}{p} \frac{d}{d t} \int|v|^{p} d x+\nu \int|\nabla v|^{2}|v|^{p-2} d x \\
& \leq C \nu\left(\int|v|^{3 p} d x\right)^{\frac{1}{3}}\left(\int|C(x, t)|^{3} d x\right)^{\frac{2}{3}} .
\end{aligned}
$$

Now Morrey's inequality in $\mathbb{R}^{3}, \int|\nabla \phi|^{2} d x \geq C\left(\int|\phi|^{6} d x\right)^{\frac{1}{3}}$ implies

$$
\int|\nabla v|^{2}|v|^{p-2} d x \geq C\left(\int|v|^{3 p} d x\right)^{\frac{1}{3}} .
$$

Therefore, there exists an absolute constant $\gamma_{p}$ such that, if

$$
\sup _{t \in I}\|C(x, t)\|_{L^{3}(d x)} \leq \gamma_{p}
$$

holds then

$$
\frac{d}{d t}\|v\|_{L^{p}(d x)} \leq 0
$$

holds on $I$ for $p \geq 2$. This should not surprise, even for large data, because $v$ is close to $u_{\left(t_{i}\right)} \circ A$ and $A$ is diffusive, close to volume-preserving. The constant $\gamma_{p}$ in condition (83) is locally bounded in $p \geq 2$. The norm $\|C(x, t)\|_{L^{3}(d x)}$ is nondimensional (invariant under dilations of space) and the conditions (83) and (76) are nondimensional (invariant under space-time dilations compatible with the equations). If (76) holds on a time interval $I$ and if

$$
\sup _{t \in I}\|\nabla \nabla \ell\|_{L^{3}(d x)} \leq c_{p}
$$


for appropriate $c_{p}$ then $C(x, t)$ is well defined on $I$, and (83) holds. Consequently, (84) holds on $I$, and we have that (76) and (85) imply

$$
V_{p}(I) \leq\left\|u\left(t_{i}\right)\right\|_{L^{p}(d x)}
$$

holds. Using the Weber formula in (67) it follows from (79) and (76) that

$$
\sup _{t \in I}\|u(t)\|_{L^{p}(d x)} \leq C(1+\epsilon)\left\|u\left(t_{i}\right)\right\|_{L^{p}(d x)}
$$

holds. This shows that the expansion can be defined using the criteria (76) and (85).

In order to do this, let us recall $([\mathbf{2 2}]$, based on [43]) that if $u \in$ $L^{\infty}\left([0, T] ; L^{2}\left(\mathbb{T}^{3}\right)\right) \cap L^{2}\left([0, T], H^{1}\left(\mathbb{T}^{3}\right)\right)$ is a Leray weak solution of the periodic three dimensional Navier-Stokes equations with initial datum in $u_{0} \in H^{1}\left(\mathbb{R}^{3}\right)$ then there exist nonempty intervals $I_{j}=\left[l_{j}, r_{j}\right), j=$ $0,1,2, \ldots$ such that $l_{0}=0, u \in L^{\infty}\left(J_{j} ; H^{1}\left(\mathbb{T}^{3}\right)\right) \cap L^{2}\left(J_{j} ; H^{2}\left(\mathbb{T}^{3}\right)\right)$ for $J_{j}=\left[l_{j}, \rho_{j}\right], \rho_{j}<r_{j}$. The intervals $I_{j}$ are maximal intervals of the form $I=[l, r)$ such that $u \in L^{\infty}\left(J ; H^{1}\left(\mathbb{T}^{3}\right)\right) \cap L^{2}\left(J ; H^{2}\left(\mathbb{T}^{3}\right)\right)$ for any $J \subset$ $I$ compact subset. The sum $\sum_{j=0}^{\infty} \sqrt{\left(r_{j}-l_{j}\right)}<\infty$. Clearly from definitions the points $r_{j}$ are singular times. If we make the assumption that (76) and (85) hold for any interval $I_{j}$ then the solution is regular. Indeed, from the inequality

$$
\left|\int(u \cdot \nabla \omega) \cdot \omega d x\right| \leq C_{p}\|u\|_{L^{p}}\|\nabla \omega\|_{L^{2}}^{1+\frac{3}{p}}\|\omega\|_{L^{2}}^{1-\frac{3}{p}}
$$

valid for $p>3$ and from the vorticity equation, it follows that

$$
\sup _{t \in I_{j}}\|\omega(t)\|_{L^{2}} \leq C\left\|\omega\left(l_{j}\right)\right\|_{L^{2}} \exp \left\{c \nu^{-\frac{p+3}{p-3}} \int_{l_{j}}^{r_{j}}\|u(s)\|_{L^{p}}^{\frac{2 p}{p-3}} d s\right\} .
$$

From (87) it follows that the right hand side is finite, and therefore $r_{j}$ is not a singular time, contradicting the maximality of the intervals. Thus the assumption implies that $j=0$ and the interval of regularity is $[0, T]$. The assumption involves a non-constructive aspect. A constructive procedure involves the group expansion. 
Theorem 4. Let $u_{0} \in H^{1}\left(\mathbb{T}^{3}\right)$ be divergence-free. Let $T>0$. Let $\epsilon>0$ be a small number. Consider nonempty intervals $\left[t_{i}, t_{i+1}\right]$ with $t_{0}=0$. On each interval $\left[t_{i}, t_{i+1}\right], i=0, \ldots$ we solve the system $(70)$ :

$$
\left\{\begin{array}{l}
D_{\nu}(u, \nabla) A=0 \\
D_{\nu}(u, \nabla) v=2 \nu C \nabla v \\
u=\mathbf{P}\left((\nabla A)^{*} v\right)
\end{array}\right.
$$

with initial condition $v(x, 0)=u_{0}$ and resetting conditions

$$
\left\{\begin{array}{l}
A\left(x, t_{i}\right)=x \\
v\left(x, t_{i}+0\right)=\left((\nabla A)^{*} v\right)\left(x, t_{i}-0\right)
\end{array}\right.
$$

The length $\tau_{i}=t_{i+1}-t_{i}$ of the $i$-th interval is determined by the requirement $\tau_{i}=\sup \{0<\tau<T\}$ where $\tau$ has the property

$$
\sup _{t \in\left[t_{i}, t_{i}+\tau\right]}\|\nabla \ell\|_{L^{\infty}\left(\mathbb{T}^{3}\right)} \leq \epsilon
$$

and

$$
\sup _{t \in\left[t_{i}, t_{i}+\tau\right]}\|\nabla \nabla \ell\|_{L^{3}\left(\mathbb{T}^{3}\right)} \leq c_{p}
$$

where $\ell(x, t)=A(x, t)-x$, and $c_{p}$ is the absolute constant of (85) with $p>3$. Then the solution of the Navier-Stokes equation with initial datum $u_{0}$ is regular on $\cup_{i}\left[t_{i}, t_{i+1}\right]$.

Remark. The theorem does not assert that $\cup_{i}\left[t_{i}, t_{i+1}\right]=[0, T]$.

Proof: In view of the previous argument, $\|v\|_{L^{p}\left(\mathbb{T}^{3}\right)}$ is a nonincreasing function of time in each of the intervals. The values of $\|u\|_{L^{p}\left(\mathbb{T}^{3}\right)}$ on each interval are hence controlled using (87) and, consequently, the values of $\|\omega\|_{L^{2}\left(\mathbb{T}^{3}\right)}$ are controlled using (88). It follows that for each closed interval $[0, t] \subset \cup_{i}\left[t_{i}, t_{i+1}\right]$ the solution of the Navier-Stokes equation has uniformly bounded $\|\omega\|_{L^{2}\left(\mathbb{T}^{3}\right)}$ and is therefore regular.

\section{References}

[1] V. I. Arnol'D And B. A. Khesin, Topological methods in hydrodynamics, in: "Annual review of fluid mechanics", Vol. 24, Annual Reviews, Palo Alto, CA, 1992, pp. 145-166.

[2] J. T. Beale, T. Kato, and A. J. MajdA, Remarks on the breakdown of smooth solutions for the 3-D Euler equations, Comm. Math. Phys. 94(1) (1984), 61-66.

[3] H. BeIrÃo DA VEIGA AND L. Berselli, On the regularizing effect of the vorticity direction in incompressible viscous flows, Differential Integral Equations 15(3) (2002), 345-356. 
[4] A. L. Bertozzi and P. Constantin, Global regularity for vortex patches, Comm. Math. Phys. 152(1) (1993), 19-28.

[5] D. ChaE, Remarks on the blow-up criterion of the three-dimensional Euler equations, Nonlinearity 18(3) (2005), 1021-1029.

[6] D. Chae, A. Córdoba, D. Córdoba, and M. A. Fontelos, Finite time singularities in a 1D model of the quasi-geostrophic equation, Adv. Math. 194(1) (2005), 203-223.

[7] D. Chat ANd H.-S. NAm, Local existence and blow-up criterion for the Boussinesq equations, Proc. Roy. Soc. Edinburgh Sect. A 127(5) (1997), 935-946.

[8] J.-Y. Chemin, "Perfect incompressible fluids", Translated from the 1995 French original by Isabelle Gallagher and Dragos Iftimie, Oxford Lecture Series in Mathematics and its Applications 14, The Clarendon Press, Oxford University Press, New York, 1998.

[9] S. Childress, G. R. Ierley, E. A. Spiegel, and W. R. Young, Blow-up of unsteady two-dimensional Euler and Navier-Stokes solutions having stagnation-point form, J. Fluid Mech. 203 (1989), $1-22$.

[10] A. J. Chorin, "Vorticity and turbulence", Applied Mathematical Sciences 103, Springer-Verlag, New York, 1994.

[11] A. J. Chorin, Numerical study of slightly viscous flow, J. Fluid Mech. 57(4) (1973), 785-796.

[12] P. Constantin, Note on loss of regularity for solutions of the 3-D incompressible Euler and related equations, Comm. Math. Phys. 104(2) (1986), 311-326.

[13] P. Constantin, Navier-Stokes equations and area of interfaces, Comm. Math. Phys. 129(2) (1990), 241-266.

[14] P. Constantin, Geometric and analytic studies in turbulence, in: "Trends and perspectives in applied mathematics", Appl. Math. Sci. 100, Springer, New York, 1994, pp. 21-54.

[15] P. Constantin, The Euler equations and nonlocal conservative Riccati equations, Math. Res. Notices 9 (2000), 455-465.

[16] P. Constantin, An Eulerian-Lagrangian approach for incompressible fluids: local theory, J. Amer. Math. Soc. 14(2) (2001), 263-278 (electronic).

[17] P. Constantin, An Eulerian-Lagrangian approach to the NavierStokes equations, Comm. Math. Phys. 216(3) (2001), 663-686.

[18] P. Constantin, Near identity transformations for the NavierStokes equations, in: "Handbook of mathematical fluid dynamics", Vol. II, North-Holland, Amsterdam, 2003, pp. 117-141. 
[19] P. Constantin, W. E, And E. Titi, Onsager's conjecture on the energy conservation for solutions of Euler's equation, Comm. Math. Phys. 165(1) (1994), 207-209.

[20] P. Constantin and C. Fefferman, Direction of vorticity and the problem of global regularity for the Navier-Stokes equations, Indiana Univ. Math. J. 42(3) (1993), 775-789.

[21] P. Constantin, C. Fefferman, and A. J. Majda, Geometric constraints on potentially singular solutions for the 3-D Euler equations, Comm. Partial Differential Equations 21(3-4) (1996), 559-571.

[22] P. Constantin and C. Foias, "Navier-Stokes equations", Chicago Lectures in Mathematics, University of Chicago Press, Chicago, IL, 1988.

[23] P. Constantin, A. J. Majda, and E. Tabak, Formation of strong fronts in the 2-D quasigeostrophic thermal active scalar, Nonlinearity 7(6) (1994), 1495-1533.

[24] P. Constantin, Q. Nie, and N. Schörghofer, Nonsingular surface quasi-geostrophic flow, Phys. Lett. A 241(3) (1998), 168-172.

[25] P. Constantin and J. Wu, The inviscid limit for non-smooth vorticity, Indiana Univ. Math. J. 45(1) (1996), 67-81.

[26] D. CóRDobA, On the geometry of solutions of the quasi-geostrophic and Euler equations, Proc. Nat. Acad. Sci. U.S.A. 94(24) (1997), 12769-12770.

[27] D. CóRdoba, Nonexistence of simple hyperbolic blow-up for the quasi-geostrophic equation, Ann. of Math. (2) 148(3) (1998), $1135-1152$.

[28] D. Córdoba and C. Fefferman, On the collapse of tubes carried by 3D incompressible flows, Comm. Math. Phys. 222(2) (2001), 293-298.

[29] D. Córdoba, C. Fefferman, and R. De la Llave, On squirt singularities in hydrodynamics, SIAM J. Math. Anal. 36(1 (2004), 204-213 (electronic).

[30] D. Córdoba, C. Fefferman, and J. L. Rodrigo, Almost sharp fronts for the surface quasi-geostrophic equation, Proc. Natl. Acad. Sci. USA 101(9) (2004), 2687-2691 (electronic).

[31] J. Deng, T. Y. Hou, ANd X. Xinwei, Improved geometric conditions for non-blowup of the 3D incompressible Euler equation, Comm. Partial Differential Equations 31(1-3) (2006), 293-306.

[32] J. Deng, T. Y. Hou, and X. Yu, Geometric properties and nonblowup of 3D incompressible Euler flow, Comm. Partial Differential Equations 30(1-3) (2005), 225-243. 
[33] W. E And C.-W. Shu, Small-scale structures in Boussinesq convection, Phys. Fluids 6(1) (1994), 49-58.

[34] D. G. Ebin and J. Marsden, Groups of diffeomorphisms and the notion of an incompressible fluid, Ann. of Math. (2) 92 (1970), $102-163$.

[35] L. Escauriaza, G. Seregin, and V. Šverák, $L_{3, \infty}$-solutions of Navier-Stokes equations and backward uniqueness, (Russian), Uspekhi Mat. Nauk 58 (2003), no. 2(350), 3-44; translation in Russian Math. Surveys 58(2) (2003), 211-250.

[36] G. L. Eyink, Energy dissipation without viscosity in ideal hydrodynamics. I. Fourier analysis and local energy transfer, Phys. D 78(3-4) (1994), 222-240.

[37] C. Foias, C. Gulllopé, and R. Temam, New a priori estimates for Navier-Stokes equations in dimension 3, Comm. Partial Differential Equations 6(3) (1981), 329-359.

[38] C. Foias and R. Temam, Gevrey class regularity for the solutions of the Navier-Stokes equations, J. Funct. Anal. 87(2) (1989), $359-369$.

[39] U. Frisch, "Turbulence", The legacy of A. N. Kolmogorov, Cambridge University Press, Cambridge, 1995.

[40] N. Ju, Geometric depletion of vortex stretch in 3D viscous incompressible flow, J. Math. Anal. Appl. 321(1) (2006), 412-425.

[41] T. Kato, Nonstationary flows of viscous and ideal fluids in $\mathbf{R}^{3}, J$. Functional Analysis 9 (1972), 296-305.

[42] P. G. Lemarié-Rieusset, "Recent developments in the NavierStokes problem", Chapman \& Hall/CRC Research Notes in Mathematics 431, Chapman \& Hall/CRC, Boca Raton, FL, 2002.

[43] J. Leray, Sur le mouvement d'un liquide visqueux emplissant l'espace, Acta Math. 63(1) (1934), 193-248.

[44] A. J. MAJdA AND A. L. BertozzI, "Vorticity and incompressible flow", Cambridge Texts in Applied Mathematics 27, Cambridge University Press, Cambridge, 2002.

[45] K. Ohkitani and P. Constantin, Numerical study of the Eulerian-Lagrangian formulation of the Navier-Stokes equations, Phys. Fluids 15(10) (2003), 3251-3254.

[46] K. Ohkitani and J. D. Gibbon, Numerical study of singularity formation in a class of Euler and Navier-Stokes flows, Phys. Fluids 12(12) (2000), 3181-3194.

[47] K. OhKITANi And M. Yamada, Inviscid and inviscid-limit behavior of a surface quasigeostrophic flow, Phys. Fluids 9(4) (1997), $876-882$. 
[48] A. Ruzmaikina and Z. Grujić, On depletion of the vortexstretching term in the 3D Navier-Stokes equations, Comm. Math. Phys. 247(3) (2004), 601-611.

[49] G. Seregin AND V. ŠverÁK, Navier-Stokes equations with lower bounds on the pressure, Arch. Ration. Mech. Anal. 163(1) (2002), $65-86$.

[50] J. SERRIN, Mathematical principles of classical fluid mechanics, in: "Handbuch der Physik" (herausgegeben von S. Flügge), Bd. 8/1, Strömungsmechanik I (Mitherausgeber C. Truesdell), SpringerVerlag, Berlin-Gttingen-Heidelberg, 1959, pp. 125-263.

[51] J. SERrin, On the interior regularity of weak solutions of the Navier-Stokes equations, Arch. Rational Mech. Anal. 9 (1962), 187-195.

[52] J. T. Stuart, Nonlinear Euler partial differential equations: singularities in their solution, in: "Applied mathematics, fluid mechanics, astrophysics" (Cambridge, MA, 1987), World Sci. Publishing, Singapore, 1988, pp. 81-95.

[53] W. Weber, Uber eine Transformation der hydrodynamischen Gleichungen, J. Reine Angew. Math. 68 (1868), 286-292.

Department of Mathematics

The University of Chicago

Chicago, IL 60637

USA

E-mail address: const@math.uchicago.edu

Rebut el 31 de gener de 2008. 